Bio - grafía. Escritos sobre la Biología y su Enseñanza. ISSN 2027-1034

Edición Extraordinaria. p.p. 319 - 329

Memorias del IX Encuentro Nacional de Experiencias en Enseñanza de la Biología y la Educación Ambiental. IV Congreso Nacional de Investigación en Enseñanza de la Biología.

\title{
EVALU ACIÓN DE PROFESORES DE BIOLOGÍA Y CIENCIAS NATURALES Y EDUCACIÓN AMBIENTAL EN FORMACIÓN; UNA REFLEXIÓN DE LOS RESULTADOS PRUEBAS SABERPRO 2014
}

\section{EVALUATION OF TEACHERS OF BIOLOGY AND NATURAL SCIENCES AND ENVIRONMENT AL EDUCATION IN TRAINING; A REFLECTION OF THE RESULTS TESTS SABERPRO 2014}

\author{
Ríos, Christian Reynel ${ }^{1}$ \\ Velásquez, Juan Nicolás ${ }^{2}$ \\ Martínez, Cristian Yasser ${ }^{3}$ \\ Mendoza, Luis Felipe ${ }^{4}$
}

\section{RESUMEN:}

La presente investigación expone algunos de los factores asociados al desempeño académico que han influenciado los resultados de la prueba SABERPRO 2014, plasmados en tres competencias específicas (Enseñar, Formar y Evaluar) de ocho programas académicos de cuatro universidades del país para contribuir a las discusiones que problematizan la evaluación relacionada a la formación docente en el área de las ciencias naturales y la educación ambiental. Mediante una investigación de métodos mixtos se ajustan modelos estadísticos y se desarrollan entrevistas semiestructuradas que amplían los análisis de los resultados de dicho modelo; por un lado posee información que lo complementa, y por otro, contiene posturas de administrativos y docentes de cada programa. Se concluye que los factores que tienen efecto tanto positivo como negativo en los resultados de la prueba son: acreditación institucional, formación máxima docente, plan de estudios y nivel socioeconómico de los estudiantes.

\section{PALABRAS CLAVES:}

Resultados de SABERPRO, factores asociados al desempeño, licenciados en biología y ciencias naturales y educación ambiental e investigación de métodos mixtos.

\footnotetext{
1 cristianrios0616@hotmail.com

2 nicolas velasquez88@hotmail.com

3 joeramo-ne93@gmail.com

4 luisfemend@gmail.com

1234 Estudiantes de Licenciatura en Biología Universidad Distrital Francisco José de Caldas. Grupo y Semillero de Investigación BER: Biología, Enseñanza y Realidades.
} 
Bio - grafía. Escritos sobre la Biología y su Enseñanza. ISSN 2027-1034

Edición Extraordinaria. p.p. 319 - 329

Memorias del IX Encuentro Nacional de Experiencias en Enseñanza de la Biología y la

Educación Ambiental. IV Congreso Nacional de Investigación en Enseñanza de la Biología.

\begin{abstract}
ABSTRAC:
The present research exposes some factors associated with academic performance that have influenced the results of the SABERPRO 2014 test, reflected in three specific competences (Teach, Form and Evaluate) of eight academic programs of four universities of the country to contribute to the discussions that they problematize the evaluation related to the formation of teacher in the area of the natural sciences and the environmental education. Through a research of mixed methods, statistical models are adjusted and semi-structured interviews are developed that extend the analyzes to the results of said model; on the one hand it has information that complements it, and on the other, it contains administrative positions and teachers of each program. It is concluded that the factors that have both positive and negative effects on the test results are: institutional accreditation, level of teacher training, composition of the curriculum and socioeconomic level of students.
\end{abstract}

KEYWORDS: SABREPRO results, factors associated with performance, biology and natural sciences and environmental education teachers and research of mixed methods.

\title{
INTRODUCCIÓN
}

Actualmente en Colombia los sujetos que buscan constituirse como profesionales mediante la educación superior al finalizar su proceso formativo deben desarrollar una evaluación institucional externa que desde el 2013 recibe el nombre de SABERPRO. Esta cuantifica los conocimientos y habilidades enmarcados dentro de determinadas competencias, incorporados durante dicho proceso. Para este caso son estudiantes de las carreras: Licenciatura en Biología, Química y Física y Licenciatura en Ciencias Naturales y Educación Ambiental de 4 universidades del país; Universidad Distrital Francisco José de Caldas, Universidad Pedagógica Nacional, Universidad Pedagógica y Tecnológica de Colombia y Universidad del Tolima. Los resultados que se obtienen de estas pruebas, dada su presunta relación con ciertos factores asociados al desempeño tales como el género, estructuración del plan de estudio, formación máxima docente, estrato socioeconómico entre otros, son ahora elemento de análisis dentro de amplias investigaciones como las de Garbanzo (2007); Montero \& Villalobos (2007); Gil, Rodríguez, Sepúlveda, Rondón \& Gómez-Restrepo (2013) y Caso-Niebla \& Hernández (2007) entre otros. De acuerdo a lo anterior, se contraponen los resultados para el módulo de competencias específicas de la prueba en el 2014 con cada factor asociado que hace presencia al interior de cada proyecto seleccionado y se fortalece el análisis mediante las posturas de docentes y directivos de los programas seleccionados respecto a la presunta relación entre los resultados de la prueba y los factores asociados desde algunos marcos teóricos.

En primera medida, en lo que a esta prueba se refiere es preciso mencionar que en Colombia es regulada por el Instituto Colombiano para el Fomento a la Educación Superior ICFES; en el año 2013 la prueba cambia su nominación de ECAES que se 


\section{Bio - grafía. Escritos sobre la Biología y su Enseñanza. ISSN 2027-1034}

Edición Extraordinaria. p.p. 319 - 329

Memorias del IX Encuentro Nacional de Experiencias en Enseñanza de la Biología y la Educación Ambiental. IV Congreso Nacional de Investigación en Enseñanza de la Biología.

aplicaba solo a algunas carreras profesionales y tecnológicas, y pasa a llamarse SABERPRO, en respuesta al Decreto 3963 del 2009, que busco dotarle de un carácter autocritico para responder problemas contextualizados; en respuesta a estos cambios, en el 2011 se realizan cambios en relación con los programas de Ciencias de la educación, Ciencias naturales e ingenierías, que empiezan a ser evaluados por competencias genéricas y específicas, mediante preguntas planteadas con base en determinados núcleos que se componen de desempeños particulares (MEN, 2014).

Antes de hacer mención a los factores asociados al desempeño es preciso aclarar que se entiende por desempeño o rendimiento académico, que para el caso son manejados como sinónimos ,se entiende como "el promedio obtenido por el alumno" (Delgado y Palos, 2007, p. 6) y "el conjunto de transformaciones que se dan en los estudiantes, no sólo en el aspecto cognoscitivo, sino también en las aptitudes, actitudes, competencias, ideales e intereses, a través del proceso enseñanza-aprendizaje que se evidencia en la manera como éstos se enfrentan en la cotidianidad a los retos que le impone su propia existencia y relación con los otros" (Quintero, Vallejo \& Giraldo, 2013, p.100). Así mismo, Garbanzo (2007), que señala al rendimiento académico como un fenómeno multicausal, plantea una serie de determinantes del desempeño académico de orden personal, social e institucional que orientan la escogencia de los factores asociados ahora estudiados, de origen sociodemográficos: género y estrato socioeconómico, e institucional: acreditación, formación máxima docente, plan de estudios, grupos de investigación y uso de los resultados. Ahora bien, mencionado lo anterior es posible preguntar ¿En qué medida factores institucionales y sociodemográficos inciden en los resultados de la evaluación de competencias específicas de la prueba SABERPRO 2014 de 8 programas de Licenciatura en el área de las Ciencias Naturales y Educación?

\section{METODOLOGÍA}

La investigación de métodos mixtos según Johnson y Onwuegbuzie (2004), citados por Pereira (2011) son el tipo de estudio donde confluyen técnicas de investigación, métodos, enfoques y conceptos expresados en términos cuantitativos o cualitativos de acuerdo al criterio del investigador. Hamui (2013) describe el modelo de investigación de métodos mixtos que ahora se presenta: el modelo de triangulación, donde datos cualitativos y cuantitativos emergen de manera simultánea, involucran una misma población y se integran en un análisis posterior. En la tabla 1 se expone la forma en que son sincronizadas en fases generales las fases de las metodologías cualitativas y cuantitativas utilizadas.

\section{Tabla 1 Sincronización metodológica}

\begin{tabular}{|l|l|lr|r|}
\hline \multicolumn{2}{|c|}{ Fases generales } & \multicolumn{2}{|c|}{ Metodología Cualitativa } & Metodología Cuantitativa \\
\hline $\begin{array}{l}\text { Recolección de la } \\
\text { información }\end{array}$ & $\begin{array}{l}\text { Determinación del } \\
\text { de estudio } \\
\text { Selección }\end{array}$ & de & la & \\
\hline
\end{tabular}


Bio - grafía. Escritos sobre la Biología y su Enseñanza. ISSN 2027-1034

Edición Extraordinaria. p.p. 319 - 329

Memorias del IX Encuentro Nacional de Experiencias en Enseñanza de la Biología y la Educación Ambiental. IV Congreso Nacional de Investigación en Enseñanza de la Biología.

\begin{tabular}{|l|l|l|}
\hline $\begin{array}{l}\text { Disposición de la } \\
\text { información }\end{array}$ & $\begin{array}{l}\text { Comunicación que será } \\
\text { estudiada }\end{array}$ & \\
\hline & $\begin{array}{l}\text { Identificación } \\
\text { Clasificación de unidades y } \\
\text { Categorización } \\
\text { Comprobación de fiabilidad } \\
\text { Disposición } \\
\text { transformación }\end{array}$ & $\begin{array}{l}\text { Presentación yanización de los datos. } \\
\text { organ }\end{array}$ \\
\hline $\begin{array}{l}\text { Manipulación y análisis } \\
\text { de la información }\end{array}$ & $\begin{array}{l}\text { Procesos para obtener } \\
\text { resultados y conclusiones. }\end{array}$ & $\begin{array}{l}\text { Tratamiento y exploración } \\
\text { de los datos. }\end{array}$ \\
\hline & Análisis mixto \\
\hline
\end{tabular}

\section{Metodología Cualitativa}

a) Recolección de la información: Además de determinar el objeto de estudio desde la selección de una dirección, comportamiento o hecho, delimitado por el tiempo, espacio, individuos y contexto que se desea analizar (Abela, 2002), se selecciona la comunicación que será estudiada, la cual se obtiene desde una entrevista semiestructurada que previamente tuvo que ser validada mediante un juicio de expertos (Escobar, 2008). Previo a su desarrollo con participación de administrativos y docentes, precisa del diligenciamiento de un consentimiento informado, elemento imprescindible dentro de la investigación cualitativa (Eisner, 1998).

b) Disposición de la información: Aquí se identifican el tipo de unidades que surgirán en el análisis al contenido de las entrevistas y se establecen las reglas para codificar, los tipos de construcción de categorías, así como el marco de categorización final. Al finalizar la categorización elaborada mediante el uso del software especializado NVIVO, se comprueba la fiabilidad, impresa desde codificaciones independientes elaboradas como mínimo de dos investigadores (Kripperdorf, 1990) citado por (Abela, 2002), y presente en los datos que permanecen estables en las posibles variaciones del proceso de análisis (Abela, 2002). Por último, se dispone la información en un conjunto organizado y abarcable para dar solución a cuestiones de la investigación y se transforma en matrices y gráficos (Revuelta \& Sánchez, 2009).

c) Manipulación y análisis de la información: Para la obtención de resultados se seleccionan las unidades de registro más relevantes de acuerdo a la descripción, interpretación, concurrencia de códigos, comparación y contextualización y en la obtención de conclusiones se consolidan teóricamente los resultados desde la aplicación de otras teorías y la síntesis con resultados de otras investigaciones (Rodríguez, Lorenzo \& Torres, 2005). 
Bio - grafía. Escritos sobre la Biología y su Enseñanza. ISSN 2027-1034

Edición Extraordinaria. p.p. 319 - 329

Memorias del IX Encuentro Nacional de Experiencias en Enseñanza de la Biología y la Educación Ambiental. IV Congreso Nacional de Investigación en Enseñanza de la Biología.

\section{Metodología Cuantitativa}

a) Recolección de la información: Aquí como lo indican Lafuente \& Marín (2008), se debe hacer una definición minuciosa de las variables dependientes e independientes. La variable dependiente, definida como el fenómeno resultante que debe ser explicado (Amiel, 2007) es el promedio de los resultados para los módulos de competencias específicas de la prueba SABERPRO 2014 y las variables independientes son la causa que da origen al fenómeno, los factores asociados (Amiel, 2007). La información recolectada y definida a manera de variable dependiente es tomada desde una base de datos FTP provista por el ICFES y validada por un experto en el área de la estadística.

b) Disposición de la información: Aquí se llevan a una hoja de cálculo los datos numéricos de las variables descritas en la fase anterior. Las variables dependientes e independientes se ubican en los ejes $X$ y $Y$ de una matriz que es llevada al software especializado SPSS para su tratamiento.

c) Manipulación y análisis de la información: Se realiza un análisis inferencial en el cual se plantean hipótesis de investigación sobre las variables independientes y su distribución normal en contraste los promedios de los módulos que son puestas a prueba mediante la prueba Shapiro-Wilk. La aceptación de determinadas hipótesis da lugar a pruebas paramétricas, donde una significación bilateral superior a 0.05 permite inferir que ese factor no tiene efecto en los resultados de la prueba; por el contrario una significación bilateral inferior a 0.05 da significancia a la correlación de Pearson que arroja un valor definitivo sobre el efecto negativo o positivo que posee cada factor sobre los resultados de la prueba. Este valor de correlación oscila entre 1 y -1 , lo cual permite observar el tipo de efecto de acuerdo al signo que acompaña al valor y su grado de fiabilidad reflejado en asteriscos $\left({ }^{*} 0^{* *}\right)$; un asterisco expone un error estimado de 0.05 y dos asteriscos un error estimado de 0.01 .

Tabla 2 Población seleccionada

\begin{tabular}{|l|l|}
\hline \multicolumn{1}{|c|}{ Programas académicos } & \multicolumn{1}{c|}{ Universidad } \\
\hline $\begin{array}{l}\text { Licenciatura en Biología (PCLB), en } \\
\text { Química (PCLQ) y en Física (PCLF) }\end{array}$ & $\begin{array}{l}\text { Universidad Distrital Francisco José de } \\
\text { Caldas }\end{array}$ \\
\hline $\begin{array}{l}\text { Licenciatura en Biología (PALB), en } \\
\text { Química (PALQ) y en Física (PALF) }\end{array}$ & Universidad Pedagógica Nacional \\
\hline $\begin{array}{l}\text { Licenciatura en Ciencias naturales y } \\
\text { educación ambiental (LCNEA) }\end{array}$ & $\begin{array}{l}\text { Universidad Pedagógica y Tecnológica de } \\
\text { Colombia }\end{array}$ \\
\hline $\begin{array}{l}\text { Licenciatura en Ciencias naturales y } \\
\text { educación ambiental (LeCNEA) }\end{array}$ & Universidad del Tolima \\
\hline
\end{tabular}


Bio - grafía. Escritos sobre la Biología y su Enseñanza. ISSN 2027-1034

Edición Extraordinaria. p.p. 319 - 329

Memorias del IX Encuentro Nacional de Experiencias en Enseñanza de la Biología y la Educación Ambiental. IV Congreso Nacional de Investigación en Enseñanza de la Biología.

En la tabla 2. se exponen los programas y las universidades escogidas para la investigación, seleccionadas por formar licenciados en el campo de las ciencias naturales y la educación ambiental, tanto en programas multidisciplinares en la UT y UPTC, y programas más específicos como los de la UDFJC y UPN.

\section{RESULTADOS Y DISCUSIÓN:}

La tabla 3 expone los promedios de los resultados de la prueba para cada uno de los proyectos seleccionados. Se observa un mejor desempeño de las carreras de formación en áreas específicas frente a las de formación multidisciplinar.

Tabla 3 Resultados de SABERPRO 2014 de 8 programas

\begin{tabular}{|c|c|c|c|c|c|c|}
\hline Universidad & Licenciatura & Muestra & Competencia & Promedio & Prom competencias & Prom. comp Univ \\
\hline \multirow{9}{*}{ UDFJC } & \multirow{3}{*}{ Lic. Biologia } & \multirow{3}{*}{73} & Enseñar & 11,16 & \multirow{3}{*}{$11,03^{* *}$} & \multirow{9}{*}{$10,73^{* * 8}$} \\
\hline & & & \begin{tabular}{|l|} 
Evaluar \\
\end{tabular} & 10,9 & & \\
\hline & & & Formar & 11,03 & & \\
\hline & \multirow{3}{*}{ Lic. Quimíca } & \multirow{3}{*}{46} & Enseñar & 11,03 & \multirow{3}{*}{10,84} & \\
\hline & & & Evaluar & 10,81 & & \\
\hline & & & Formar & 10,69 & & \\
\hline & \multirow{3}{*}{ Lic. Fisica } & \multirow{3}{*}{98} & Enseñar & 10,33 & \multirow{3}{*}{10,33} & \\
\hline & & & \begin{tabular}{|l|} 
Evaluar \\
\end{tabular} & 10,37 & & \\
\hline & & & Formar & 10,29 & & \\
\hline \multirow{9}{*}{ UPN } & \multirow{3}{*}{ Lic. Biologia } & \multirow{3}{*}{$104^{*}$} & Enseñar & 10,96 & \multirow{3}{*}{10,87} & \multirow{9}{*}{$10,77^{* * *}$} \\
\hline & & & Evaluar & 10,82 & & \\
\hline & & & Formar & 10,84 & & \\
\hline & \multirow{3}{*}{ Lic. Quimíca } & \multirow{3}{*}{64} & Enseñar & 10,94 & \multirow{3}{*}{10,92} & \\
\hline & & & \begin{tabular}{|l|} 
Evaluar \\
\end{tabular} & 10,87 & & \\
\hline & & & Formar & 10,96 & & \\
\hline & \multirow{3}{*}{ Lic. Fisica } & \multirow{3}{*}{44} & Enseñar & 10,5 & \multirow{3}{*}{10,53} & \\
\hline & & & Evaluar & 10,52 & & \\
\hline & & & Formar & 10,59 & & \\
\hline \multirow{3}{*}{ UPTC } & \multirow{3}{*}{$\begin{array}{l}\text { Lic. Ciencias Naturales y } \\
\text { Edu. Ambiental }\end{array}$} & \multirow{3}{*}{35} & Enseñar & 10,24 & \multirow{3}{*}{10,32} & \multirow{3}{*}{$10,32^{* * *}$} \\
\hline & & & \begin{tabular}{|l|} 
Evaluar \\
\end{tabular} & 10,4 & & \\
\hline & & & Formar & 10,34 & & \\
\hline \multirow{3}{*}{ UT } & Lic. en educación basica & \multirow{3}{*}{$30^{*}$} & Enseñar & 7,7 & \multirow{3}{*}{$7,83^{* *}$} & \multirow{3}{*}{$7,83^{* * *}$} \\
\hline & con enfasis Ciencias & & Evaluar & 8,6 & & \\
\hline & Naturales v Educación & & Formar & 7.2 & & \\
\hline
\end{tabular}

La tabla 4 muestra los diferentes factores, la significancia bilateral, los valores para la correlación de Pearson y las unidades de registro con mayor potencial interpretativo sobre la relación entre los factores asociados y el desarrollo de conocimiento y habilidades relativas a cada competencia específica que se refleja en un promedio general.

Tabla 4 Resultados cuantitativos y cualitativos

\begin{tabular}{|l|l|l|l|}
\hline $\begin{array}{c}\text { Factor } \\
\text { asociado }\end{array}$ & $\begin{array}{l}\text { Significan } \\
\text { cia } \\
\text { bilateral }\end{array}$ & $\begin{array}{l}\text { Correlac } \\
\text { ión de } \\
\text { Pearson }\end{array}$ & \multicolumn{1}{|c|}{ Unidad de registro } \\
\hline Género & .842 & $\mathrm{XXX}$ & $\begin{array}{l}\text { "No, definitivamente no. Yo no puedo generalizar } \\
\text { Porque aquí tenemos chicas que se han } \\
\text { destacado con la producción académica y un } \\
\text { liderazgo académico fuerte y de igual manera los } \\
\text { niños, o sea, no podemos decir sí PALF_UPN. }\end{array}$ \\
\hline $\begin{array}{l}\text { Estrato } \\
\text { socioecon }\end{array}$ & .001 & $-.644^{* *}$ & $\begin{array}{l}\text { "Para yo poder evaluar y para sentirme evaluado } \\
\text { tengo que tener como una posición de }\end{array}$ \\
\hline
\end{tabular}


Bio - grafía. Escritos sobre la Biología y su Enseñanza. ISSN 2027-1034

Edición Extraordinaria. p.p. 319 - 329

Memorias del IX Encuentro Nacional de Experiencias en Enseñanza de la Biología y la Educación Ambiental. IV Congreso Nacional de Investigación en Enseñanza de la Biología.

\begin{tabular}{|c|c|c|c|}
\hline ómico (4) & & & $\begin{array}{l}\text { preparación, sea el tema que sea. Y hay } \\
\text { personas que viven en un entorno tan agresivo } \\
\text { tan violento que no les gusta sentir esas } \\
\text { presiones" PALQ_UPN. }\end{array}$ \\
\hline $\begin{array}{l}\text { Plan de } \\
\text { estudios } \\
\text { (Electivas) }\end{array}$ & .001 & $.613^{* *}$ & $\begin{array}{l}\text { "Uno encuentra planes de estudio en donde hay } \\
\text { competencias para } 1 \text { mes, desde mi perspectiva } \\
\text { la competencia fundamental como evaluar, eso } \\
\text { no lo adquiere un estudiante en } 1 \text { mes, eso no lo } \\
\text { adquiere en una asignatura que se llame } \\
\text { "evaluación educativa" LeCNEA_UT. }\end{array}$ \\
\hline $\begin{array}{l}\text { Grupos de } \\
\text { investigaci } \\
\text { ón } \\
\text { categoriza } \\
\text { dos en } \\
\text { COLCIENC } \\
\text { IAS }\end{array}$ & .223 & $x x x$ & $\begin{array}{l}\text { "Claro incluso nosotros hacíamos un análisis } \\
\text { interesante de algunos años y los chicos que } \\
\text { hacen parte de los grupos de investigación pues } \\
\text { tenían mejores resultados en tanto diferentes } \\
\text { competencias" PALF_UPN }\end{array}$ \\
\hline $\begin{array}{l}\text { Formación } \\
\text { máxima } \\
\text { docente } \\
\text { (Especializ } \\
\text { ación) }\end{array}$ & .001 & $-.626^{* *}$ & $\begin{array}{l}\text { "Obviamente hay una influencia en ese proceso } \\
\text { del nivel de formación de los maestros" } \\
\text { LeCNEA_UT. }\end{array}$ \\
\hline $\begin{array}{l}\text { Uso de } \\
\text { resultados }\end{array}$ & .801 & $x x x$ & $\begin{array}{l}\text { "tú me estas preguntando si relacionamos la } \\
\text { prueba SABER PRO como los resultados y } \\
\text { fortalecimiento para mejorar estos espacios, } \\
\text { entonces allí no lo hemos hecho directamente, a } \\
\text { no ser que los profesores que dirigen estos } \\
\text { espacios lo tengan en cuenta, pero no tendría } \\
\text { conocimiento al respecto" PALQ_UPN }\end{array}$ \\
\hline $\begin{array}{l}\text { Acreditaci } \\
\text { ón }\end{array}$ & .001 & $.943^{\star \star}$ & $\begin{array}{l}\text { "Si, si, a mí me parece excepcional, que es } \\
\text { dispendioso los procesos administrativos y } \\
\text { muchas veces quitan tiempo para la academia } \\
\text { para que el profesor se forme, para que el } \\
\text { profesor investigue. Pero yo si pienso que en } \\
\text { entidades o instituciones pequeñas mejoran los } \\
\text { procesos, en todo, en gestión, en academia, } \\
\text { pienso yo que sí.". PALQ_UPN }\end{array}$ \\
\hline
\end{tabular}


Bio - grafía. Escritos sobre la Biología y su Enseñanza. ISSN 2027-1034

Edición Extraordinaria. p.p. 319 - 329

Memorias del IX Encuentro Nacional de Experiencias en Enseñanza de la Biología y la

Educación Ambiental. IV Congreso Nacional de Investigación en Enseñanza de la Biología.

\begin{abstract}
Análisis mixto
Género: Papadópulos \& Radakovich (2006) exaltan los avances que se han hecho en las últimas décadas en América Latina y el Caribe para disminuir de desigualdad de género en el acceso, permanencia y egreso en la educación superior. Esto coincide con los datos cuantitativos e información cualitativa obtenida, pues en primera medida el índice de significación para las variables Masculino y Femenino supera los ,005 lo que significa que estadísticamente no existe relación entre género y los resultados de competencias específicas de la prueba S ABER PRO 2014. Por otra parte desde el análisis cualitativo se señala en varios registros que el género de los estudiantes no es un factor que incida en el desempeño académico de los estudiantes.
\end{abstract}

Estrato: Las políticas de equidad ejecutadas institucionalmente para hacerle frente las posibles dificultades económicas que puede afrontar los estudiantes se centra en los estudiantes de estratos bajos (Moya, 2011). Mediante el análisis cuantitativo se muestra a Estudiantes estrato 4 como un factor negativo fuerte, con un índice de Correlación de Pearson de,$- 644^{\star *}$. Si tomamos en cuenta lo anteriormente descrito y se contrasta con algunas unidades de registro es posible observar como efectivamente se enfatiza en atender a estudiantes provenientes de poblaciones vulnerables, haciendo claridad que las cuatro universidades seleccionadas son de carácter público.

Plan de estudios: Este factor desde la variable Créditos a electivas presenta un índice de correlación de Pearson de, $613^{\star \star}$, lo que reafirma el efecto positivo que posee sobre el promedio de los resultados del módulo de competencias específicas. Se observa que las universidades que destinaron un mayor porcentaje de créditos para materias electivas dentro de su plan de estudios mostraron mejores resultados, esto se debe a que estas deben ser programadas y ofertadas con base a estudios y análisis previos de las del mercado laboral (Guerrero, 2013). El mismo autor señala algunas de las posibilidades que ofrece este factor tales como: reforzar aspectos disciplinares de la profesión, actualizándolos para así responder a problemáticas del contexto, lo cual concuerda con la noción de competencias que propone por ejemplo Rey (1996).

Grupos de investigación categorizados por Colciencias: Como se observa en la tabla 4, la significancia bilateral es superior a .005, por lo cual se descarta el análisis de correlación de Pearson. Este valor puede estar determinado por la homogeneidad de los datos, pues los valores para cada una de las variables de este factor se repiten. Esto se contrapone a las posturas de algunos programas que señalan los conocimientos y capacidades con que se constituye un estudiante dentro de un grupo de investigación y cómo esto influye positivamente en los resultados de la prueba.

Formación máxima docente: Para la variable de especialización se obtiene un valor desde el modelo de correlación de Pearson $-0.626^{\star \star}$ que muestra su efecto negativo en los resultados de la prueba. Esto aparece contrapuesto a las posturas de (Portilla) 2002, según el cual, los estudiantes instruidos por docentes sin este tipo de estudios, es decir, sin estudios de posgrado, son los que muestran resultados más bajos; para complementar, lo propuesto por Portilla (2002) coincide con lo planteado por algunos 
Bio - grafía. Escritos sobre la Biología y su Enseñanza. ISSN 2027-1034

Edición Extraordinaria. p.p. 319 - 329

Memorias del IX Encuentro Nacional de Experiencias en Enseñanza de la Biología y la Educación Ambiental. IV Congreso Nacional de Investigación en Enseñanza de la Biología.

programas, que identifican la relación entre un mayor nivel de formación del cuerpo docente y el desempeño académico.

Uso de resultados: Este factor presenta una significación bilateral superior a 0,05 por lo cual se infiere que este factor no influye en el resultado de los módulos de competencias específicas de la prueba SABERPRO 2014. Por otro lado, en concordancia con lo propuesto por Gil, Rodríguez, Sepúlveda, Rondón \& Gómez-Restrepo (2013) y lo expuesto por algunos proyectos, la manera en que se hace uso de estos resultados es con fines netamente comparativos. Además estos no influyen en la ejecución de procesos institucionales relacionados a la formación docente. Esto deja ver las posibles fracturas presentes entre la formación de licenciados en el área de las ciencias naturales y la evaluación por competencias que se les es aplicada a los individuos que se forman en estas.

Acreditación: Con una correlación de Pearson que arroja un .943 ${ }^{\star \star}$ como valor para este factor, se infiere que este es el que mayor efecto positivo presenta sobre los resultados de la prueba en el módulo de competencias específicas. Esto coincide con las posturas emitidas desde la mayoría de los proyectos entrevistados, plasmadas en unidades de registro como la señalada en la tabla 4 y puede encontrar en explicación en lo planteado por Borroto y Salas (2004), quienes identifican a la acreditación universitaria como el acto legal por el cual una institución educativa certifica que los procesos de formación que se llevan a cabo al interior de esta pueden responder a evaluaciones externas que miden los niveles de calidad de la educación que allí se imparte. En complemento, el programa que a esa fecha no se encontraba acreditado es el que presenta los promedios más bajos.

\section{CONCLUSIONES}

El factor asociado de origen sociodemográfico que tiene efecto en los resultados de competencias específicas en la prueba SABERPRO para futuros licenciados en el área de las ciencias naturales y educción ambiental es el Estrato sociodemográfico, que en su variable ESTRATO_4 influye de forma negativa.

Los factores de origen institucional que tienen efecto positivo en los resultados de competencias específicas de la prueba SABERPRO son: la acreditación y el plan de estudios en su variable ELECTIVAS. Por el contrario, el factor de mismo origen que tiene efecto negativo en los resultados de la prueba es la Formación máxima docente en su variable de ESPECIALIZACIÓN.

La formación de docentes en el área de las ciencias naturales en Colombia parece distar de pruebas como la SABERPRO que se proponen con base en un marco educativo internacional, como la educación y evaluación por competencias, pudiendo llegar a desconocer las particularidades, objetivos y necesidades que hacen presencia al interior de cada programa académico abocada a este tipo de formación. 
Bio - grafía. Escritos sobre la Biología y su Enseñanza. ISSN 2027-1034

Edición Extraordinaria. p.p. 319 - 329

Memorias del IX Encuentro Nacional de Experiencias en Enseñanza de la Biología y la Educación Ambiental. IV Congreso Nacional de Investigación en Enseñanza de la Biología.

\section{REFERENCIAS BIBLIOGRÁFICAS}

Abela, J. A. (2002). Las técnicas de análisis de contenido: una revisión actualizada.

Amiel Pérez, J. (2007). Las variables en el método científico. Revista de la Sociedad Química del Perú, 73(3), 171-177.

Borroto Cruz, E. R., \& Salas Perea, R. S. (2004). Acreditación y evaluación universitarias. Educación Médica Superior, 18(3), 1-1.Casanova, M. A. (2007). Evaluación: Concepto, tipología y objetivos. España: La Muralla.

Caso-Niebla, J., \& Hernández, L. (2007). Variables que inciden en el rendimiento académico de adolescentes mexicanos. Revista latinoamericana de psicología, 39(3), 487-501.

Decreto 3963. Ministerio de Educación Nacional. Bogotá, Colombia, 14 de Octubre 2009.

Delgado, P., Raúl, J., \& Palos, P. A. (2007). Desempeño académico y conductas de riesgo en adolescentes. Revista de educación y desarrollo, 7, 5.

Escobar-Pérez, J., \& Cuervo-Martínez, A. (2008). Validez de contenido y juicio de expertos: una aproximación a su utilización. Avances en medición, 6(1), 27-36.

Eisner, E. W. (1998). Ojo llustrado. Paidós.

Garbanzo, G. M. (2007). Factores asociados al rendimiento académico en estudiantes universitarios, una reflexión desde la calidad de la educación superior pública. Educación, 31(1).

Gil, F. A., Rodríguez, V. A., Sepúlveda, L. A., Rondón, M. A., \& Gómez-Restrepo, C. (2013). Impacto de las facultades de medicina y de los estudiantes sobre los resultados en la prueba nacional de calidad de la educación superior (SABER PRO). Revista Colombiana de Anestesiología, 41(3), 196-204.

Guerrero, M. F. C. (2013). Las asignaturas optativas de formación profesional en la planificación curricular de pregrado. Universitas, (18).

Hamui-Sutton, A. (2013). Un acercamiento a los métodos mixtos de investigación en educación médica. Investigación en educación médica, 2(8), 211-216.

Ministerio de Educación Nacional, (2014). LINEAMIENTOS DE CALIDAD PARA LAS LICENCIATURAS EN EDUCAC IÓN (Programas de Formación Inicial de Maestros). Bogotá, pp.1 - 37.

Lafuente lbáñez, C., \& Marín Egoscozábal, A. (2008). Metodologías de la investigación en las ciencias sociales: Fases, fuentes y selección de técnicas. Revista escuela de administración de negocios, (64).

Montero Rojas, E., \& Villalobos Palma, J. (2007). Factores institucionales, pedagógicos, psicosociales y sociodemográficos asociados al rendimiento académico en la Universidad de Costa Rica: Un análisis multinivel. 
Bio - grafía. Escritos sobre la Biología y su Enseñanza. ISSN 2027-1034

Edición Extraordinaria. p.p. 319 - 329

Memorias del IX Encuentro Nacional de Experiencias en Enseñanza de la Biología y la Educación Ambiental. IV Congreso Nacional de Investigación en Enseñanza de la Biología.

Moya, C. (2011). Equidad en el acceso a la educación superior: los" cupos de equidad" en la Facultad de Ciencias Sociales de la Universidad de Chile. Calidad en la educación, (35), 255-275.

Papadópulos, J., \& Radakovich, R. (2006). Educación superior y género en América Latina y el Caribe. Sitio en Internet) Disponible en http//www. cse. cl Salazar, José Miguel (2005) Educación

Pereira Pérez, Z. (2011). Los diseños de método mixto en la investigación en educación: Una experiencia concreta. Revista Electrónica Educare, 15(1).

Portilla, A. (2002). La formación docente del profesorado universitario: perfil y líneas de formación. Ballaterra, España: Universidad Autónoma de Barcelona. Recuperado el, 15.

Quintero, M. T. Q., Vallejo, G. M. O., \& Giraldo, L. E. P. (2013). El desempeño académico: una opción para la cualificación de las instituciones educativas. Plumilla Educativa, (12), 93-115.

Rey, B. (1996). Las competencias transversales en cuestión. Paris. ESF. Traducción de Alejandro Madrid Zan. En:

http://www.philosophia.cl/biblioteca/Rey/Competencias\%20transversales.pdf

Rodríguez Sabiote, C., Lorenzo Quiles, O., \& Herrera Torres, L. (2005). Teoría y práctica del análisis de datos cualitativos. Proceso general y criterios de calidad. Revista Internacional de Ciencias Sociales y Humanidades, SOCIOTAM, 15(2). 